\title{
Visceral and ectopic fat, atherosclerosis, and cardiometabolic @ disease: a position statement
}

\author{
lan J Neeland, Robert Ross, Jean-Pierre Després, Yuji Matsuzawa, Shizuya Yamashita, Iris Shai, Jaap Seidell, Paolo Magni, Raul D Santos, \\ Benoit Arsenault, Ada Cuevas, Frank B Hu, Bruce Griffin, Alberto Zambon, Philip Barter, Jean-Charles Fruchart, Robert H Eckel, for the \\ International Atherosclerosis Society and the International Chair on Cardiometabolic Risk Working Group on Visceral Obesity
}

Findings from epidemiological studies over the past 30 years have shown that visceral adipose tissue, accurately measured by CT or MRI, is an independent risk marker of cardiovascular and metabolic morbidity and mortality. Emerging evidence also suggests that ectopic fat deposition, including hepatic and epicardial fat, might contribute to increased atherosclerosis and cardiometabolic risk. This joint position statement from the International Atherosclerosis Society and the International Chair on Cardiometabolic Risk Working Group on Visceral Obesity summarises the evidence for visceral adiposity and ectopic fat as emerging risk factors for type 2 diabetes, atherosclerosis, and cardiovascular disease, with a focus on practical recommendations for health professionals and future directions for research and clinical practice. We discuss the measurement of visceral and ectopic fat, pathophysiology and contribution to adverse health outcomes, response to treatment, and lessons from a public health programme targeting visceral and ectopic fat. We identify knowledge gaps and note the need to develop simple, clinically applicable tools to be able to monitor changes in visceral and ectopic fat over time. Finally, we recognise the need for public health messaging to focus on visceral and ectopic fat in addition to excess bodyweight to better combat the growing epidemic of obesity worldwide.

\section{Introduction}

Since 1980, the prevalence of obesity has doubled in more than 70 countries and continues to increase worldwide. ${ }^{1}$ In the USA alone, the age-adjusted prevalence of obesity (defined by BMI $\geq 30 \mathrm{~kg} / \mathrm{m}^{2}$ was estimated to be $38 \%$ (35\% of men and $40 \%$ of women) in 2014 , and about $8 \%$ had class III obesity ( $6 \%$ of men and $10 \%$ of women), the most severe form of the disorder (defined as BMI $\left.\geq 40 \mathrm{~kg} / \mathrm{m}^{2}\right)^{2}{ }^{2}$ However, although BMI might be a convenient and simple index to monitor the growth in obesity prevalence at the population level, studies have shown that obesity defined by BMI is remarkably heterogeneous $^{3}$ and that people with similar bodyweight or BMI values can have substantially different comorbidities and levels of health risk. ${ }^{4}$ Moreover, although a substantial minority of individuals with obesity have no or few cardiometabolic risk factors, ${ }^{5}$ the concept of metabolically healthy obesity is largely determined by which cutoffs are used to define abnormal risk factors. ${ }^{6}$ Furthermore, studies have shown that almost half of adults with so-called metabolically healthy obesity will develop metabolic syndrome and have higher odds for future cardiovascular disease. ${ }^{7}$ Even though the limitations of BMI as an index of obesity have been known for decades, and newer, more specific measures of adiposity-related risk have been developed, many obesity guidelines ${ }^{8-12}$ continue to recommend BMI as the optimal tool to measure obesity-related morbidity and mortality risk.

The inability of BMI to fully capture cardiometabolic risk is partly related to the fact that BMI in isolation is an insufficient biomarker of total body, and, more importantly, central abdominal fat mass and does not account for the extreme variation in intra-abdominal (visceral) fat distribution between individuals. ${ }^{13}$ For example, figure 1 shows T1-weighted MRI coronal abdominal images in two individuals, one near-normal bodyweight (BMI $25 \mathrm{~kg} / \mathrm{m}^{2}$ ) and one with obesity (BMI $30 \mathrm{~kg} / \mathrm{m}^{2}$ ). Here, BMI misrepresents the burden of visceral adipose tissue (VAT) between the two individuals. Several large cardiometabolic imaging studies, using CT or MRI, have shown that abdominal fat depots are heterogeneous and differentially associated with atherosclerosis and cardiometabolic risk..$^{14,15}$ Data from several epidemiological studies over the past 30 years have shown that VAT is an independent marker of morbidity and mortality, and abdominal subcutaneous adipose tissue (SAT) accumulation is a much weaker indicator of cardiovascular risk. ${ }^{16}$ Emerging evidence also suggests that ectopic fat deposition, including hepatic and epicardial fat linked with increased VAT deposition, might also contribute to increased atherosclerosis and cardiometabolic risk. ${ }^{17}$

On April 24, 2017, the International Atherosclerosis Society and International Chair on Cardiometabolic Risk Working Group on Visceral Obesity convened in Prague, Czech Republic, to discuss the importance of visceral adiposity and ectopic fat as an emerging risk factor for premature atherosclerosis and cardiovascular disease. The group agreed to work on the development of consensus documents, which would reflect the position of the two organisations. In this position statement, we summarise the evidence for visceral adiposity and ectopic fat as emerging risk factors for type 2 diabetes, atherosclerosis, and cardiovascular disease, with a focus on practical recommendations for health professionals and future directions for research and clinical practice.

\section{Measurement of visceral and ectopic fat}

The development of medical imaging has been a remarkable advance that has revolutionised the study of human body composition, including visceral fat. ${ }^{18-20}$ Cross-sectional
Lancet Diabetes Endocrino 2019; 7: 715-25 Published Online July 10, 2019 http://dx.doi.org/10.1016/ S2213-8587(19)30084-1 Department of Internal Medicine, Division of Cardiology, University of Texas Southwestern Medical Center, Dallas, TX, USA

(Prof IJ Neeland MD); School of Medicine, Division of Endocrinology and Metabolism, Queen's University, Kingston, ON, Canada (Prof R Ross PhD). Quebec Heart and Lung Institute Research Centre, Quebec City, QC, Canada (ProfJ-P Després PhD, Prof B Arsenault PhD); Department of Kinesiology (ProfJ-P Després), Department of Medicine (Prof B Arsenault), Faculty of Medicine, Université Laval, Quebec City, QC, Canada; Department of Endocrinology and Metabolism, Sumitomo Hospital, Osaka, Japan (ProfY Matsuzawa MD); Departments of Cardiovascular Medicine and Community Medicine, Osaka University Graduate School of Medicine, Osaka, Japan (Prof S Yamashita MD); Faculty of Health Sciences, Ben-Gurion University of the Negev, Beer-Sheva, Israel (Prof I Shai PhD); Department of Health Sciences and the EMGO Institute for Health and Care Research, VU University Amsterdam, Amsterdam, Netherlands (Prof J Seidell PhD); Department of Pharmacological and Biomolecular Sciences, Universita' degli Studi di Milano, Milan, Italy (Prof P Magni MD); Lipid Clinic Heart Institute, University of São Paulo, Medical School Hospital and Hospital Israelita Albert Einstein, Sao Paulo, Brazil (Prof R D Santos MD); Department of Clinical Nutrition and Metabolism, Clínica Las Condes, Santiago, Chile (Prof A Cuevas MD); Departments of Nutrition and Epidemiology, Harvard TH Chan 

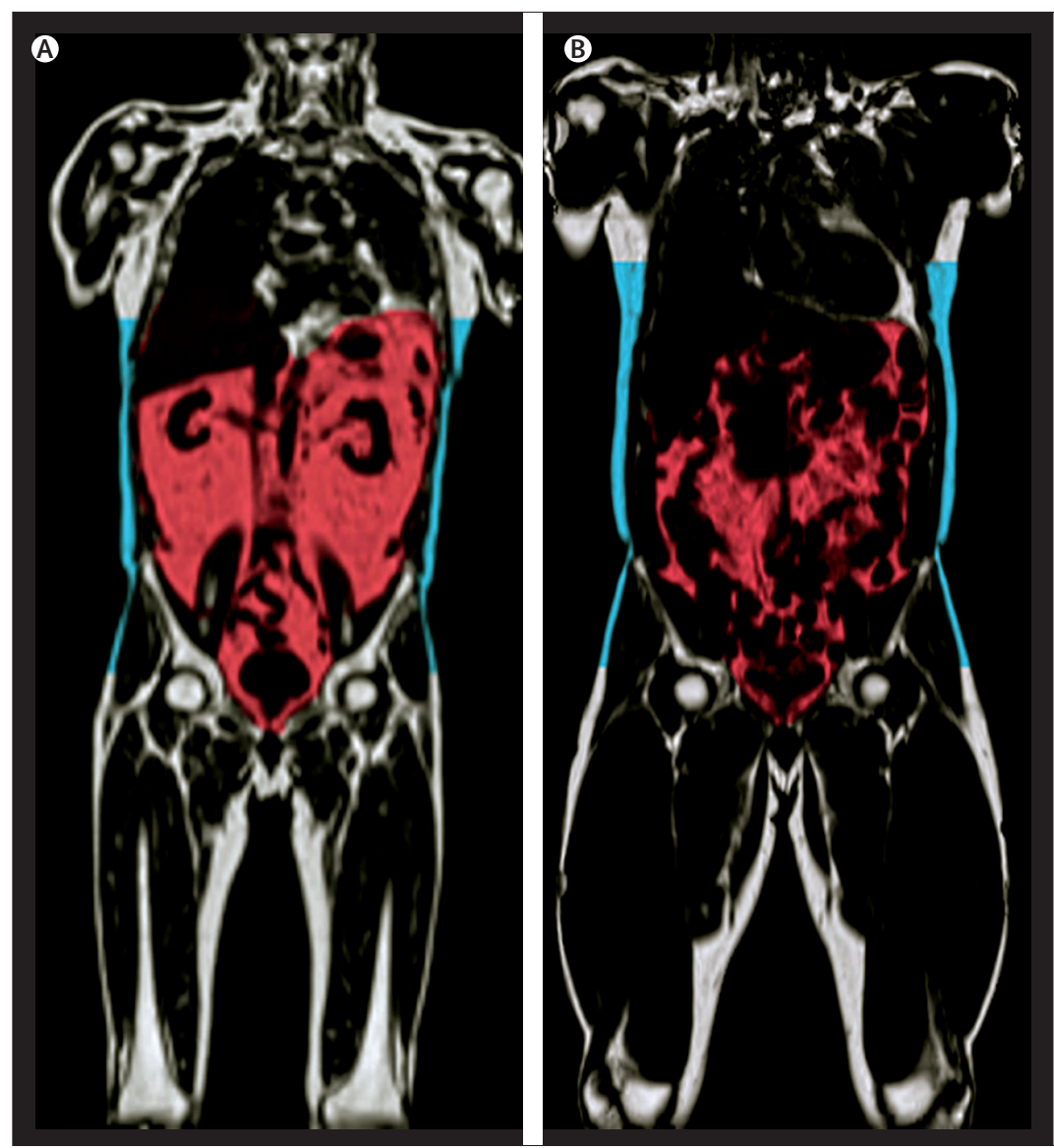

Figure 1: Variation in abdominal fat distribution

T1-weighted coronal neck-to-knee magnetic resonance images showing variation in visceral fat (red) and abdominal subcutaneous fat (blue). (A) A 67-year-old man with near-normal weight, BMI $25 \mathrm{~kg} / \mathrm{m}^{2}$, and visceral fat $2.58 \mathrm{~L} / \mathrm{m}^{2}$. (B) A 53-year-old man with obesity, BMI $30 \mathrm{~kg} / \mathrm{m}^{2}$, and visceral fat $0.88 \mathrm{~L} / \mathrm{m}^{2}$. Images from AMRA Medical

(Linköping, Sweden), reproduced from Neeland and colleagues, ${ }^{13}$ by permission of Lippincott Williams \& Wilkins.

School of Public Health, Boston, MA, USA (ProfF B Hu MD); Department of Nutritional Sciences, University of Surrey, Guildford, UK (Prof Bruce Griffin PhD); Department of Medicine, University of Padua, Padova, Italy (Prof A Zambon MD); School of Medical Sciences, University of New South Wales, Sydney, NSW, Australia (Prof P Barter MBBS); Fondation Cœur et Artères, Lille, France (ProfJ-C Fruchart PhD); and Division of Endocrinology, Metabolism and Diabetes, University of Colorado School of Medicine, Aurora, CO, USA (Prof R H Eckel MD) images of the body derived by CT or MRI can be generated at any level and on the basis of differences in imaging parameters of body fat, muscles, and bones, precise measurements of total and regional body composition are possible. Apart from quantifying adipose tissue depots, CT and MRI have been used to measure the quality of lean (skeletal muscle) ${ }^{21}$ and adipose tissues. ${ }^{22}$ Furthermore, techniques with improved spatial resolution have enabled detection and measurement of fat in areas of the body where fat is not physiologically stored, such as the liver, pancreas, heart, and skeletal muscle ${ }^{13}$ - termed ectopic fat depots. With the availability of imaging techniques, exploration of the association between these ectopic fat depots and cardiometabolic risk factors and outcomes has been possible. ${ }^{23,24}$ These methods have allowed investigators to identify the clinical importance of lipid accumulation in several tissues, including liver, skeletal muscle, and adipose tissue. ${ }^{25}$ Advances in dual-energy x-ray absorptiometry (DEXA) suggest that this method provides acceptable estimates of total and abdominal fat distribution, including visceral fat. The DEXA method for measuring visceral fat has been validated against direct measures using $\mathrm{CT}^{26}$ and $\mathrm{MRI}^{27}$ (with $R^{2}$ ranging between 0.82 and $0 \cdot 96)$. However, unlike CT or MRI, DEXA estimates VAT on the basis of the difference between total and subcutaneous abdominal fat rather than measuring it directly. Furthermore, DEXA might slightly underestimate VAT among normal-weight individuals and overestimate it among those with very severe obesity.7 Nevertheless, access to DEXA presents a feasible alternative to CT or MRI for quantifying visceral fat and practitioners are encouraged to consider this option to help to characterise the high-risk obesity phenotype.

Since routine access to CT, MRI, or DEXA might not be feasible for many clinicians and the use of these modalities for imaging of visceral and ectopic fat has historically been reserved for research purposes, several surrogate anthropometric indices (whether in isolation or combined with simple laboratory tests) of visceral fat have been developed (table). Perhaps the most widely used and accepted of these measurements is waist circumference. Ashwell and colleagues ${ }^{35}$ were the first to show a correlation between the amount of visceral fat and the waist-to-hip ratio. However, compared with the waist-to-hip ratio, waist circumference alone was shown to be more strongly associated with the amount of visceral fat. ${ }^{36}$ In a large international CT imaging study (INSPIRE ME IAA), an increased waist circumference within each unit of BMI was predictive of an increased amount of VAT. ${ }^{37}$ In view of the frequent coexistence of excess visceral and liver fat, the combined presence of an increased waist circumference and increased triglyceride levels has been associated with a high probability (around 80\%) of increased VAT levels..$^{33}$ These findings have been confirmed in other studies examining the so-called hypertriglyceridemic waist phenotype as a simple tool to screen for the presence of excess VAT and ectopic fat. ${ }^{34}$

VAT is not a routine measure in clinical settings. Therefore, the extent to which an increased VAT could affect the waist circumference threshold for increased cardiometabolic risk, and whether reductions in waist circumferenceareassociated with correspondingreductions in VAT, are important issues. In the USA, many clinicians and researchers have accepted a single threshold for adults (102 $\mathrm{cm}$ in men and $88 \mathrm{~cm}$ in women) to denote a high waist circumference, irrespective of BMI. ${ }^{38}$ However, different ethnicity-specific cutoff values for waist circumference (used in the diagnosis of the metabolic syndrome) have been proposed by the International Diabetes Federation (eg, $94 \mathrm{~cm}$ for European men and $80 \mathrm{~cm}$ for European women, and $85 \mathrm{~cm}$ for Japanese men and $90 \mathrm{~cm}$ for Japanese women). ${ }^{39}$ Although evidence including meta-analyses suggests an association between reductions in waist circumference and $\mathrm{VAT}^{40-42}$ the variance explained is fairly small (about $40 \%)^{43-45}$ However, to our knowledge there is no study reporting that a reduction in waist circumference is not associated with a corresponding reduction in VAT. Thus, although it is reasonable to 


\begin{tabular}{|c|c|c|c|}
\hline & $\begin{array}{l}\text { Correlation with } \\
\text { VAT }\end{array}$ & Advantages & Disadvantages \\
\hline BMI & $0.67-0.84^{28,29}$ & $\begin{array}{l}\text { Simple to use in the clinic; well documented in } \\
\text { the medical literature }\end{array}$ & $\begin{array}{l}\text { Fairly low correlation with VAT, especially among certain } \\
\text { racial or ethnic groups }\end{array}$ \\
\hline Waist circumference & $0.50-0.87^{28-31}$ & $\begin{array}{l}\text { Simple to use in the clinic; well documented in } \\
\text { the medical literature }\end{array}$ & $\begin{array}{l}\text { No consensus on method of measurement; variability } \\
\text { depending on location of measurement }\end{array}$ \\
\hline $\begin{array}{l}\text { Waist-to-height ratio } \\
\text { (index of central obesity) }\end{array}$ & $0.52-0 \cdot 81^{28,30}$ & $\begin{array}{l}\text { Simple to use in the clinic; well documented in } \\
\text { the medical literature }\end{array}$ & $\begin{array}{l}\text { Does not significantly improve assessment of visceral } \\
\text { adiposity beyond using waist circumference alone }\end{array}$ \\
\hline Waist-to-hip ratio & $0 \cdot 67-0.71^{29}$ & $\begin{array}{l}\text { Simple to use in the clinic; well documented in } \\
\text { the medical literature }\end{array}$ & $\begin{array}{l}\text { Does not significantly improve assessment of visceral } \\
\text { adiposity beyond using waist circumference alone }\end{array}$ \\
\hline Neck circumference & $0 \cdot 63-0.82^{31.32}$ & Simple to use in the clinic & Not very well documented in the medical literature \\
\hline Sagittal abdominal diameter* & $0.80-0.87^{29}$ & $\begin{array}{l}\text { Similar correlation with VAT as waist } \\
\text { circumference; associated with cardiometabolic } \\
\text { risk markers; can also be measured with CT }\end{array}$ & Difficult to implement in clinic \\
\hline Hypertriglyceridemic waist $\dagger$ & NA $\ddagger$ & $\begin{array}{l}\text { Integrated index including evidence of } \\
\text { metabolic dysfunction }\end{array}$ & Additional laboratory measurement required \\
\hline \multicolumn{4}{|c|}{$\begin{array}{l}\text { Data are Pearson's R. VAT=visceral adipose tissue. *Sagittal abdominal diameter is defined as the abdominal height measured as a line extending from skin to skin through } \\
\text { the centre of the abdomen in the anterior-posterior direction. }+ \text { Hypertriglyceridemic waist is defined by the presence of increased waist circumference }(\geq 90 \mathrm{~cm} \text { in men and } \\
\geq 85 \mathrm{~cm} \text { in women) accompanied by hypertriglyceridemia ( } \geq 2 \mathrm{mmol} / \mathrm{L} \text { in men and } \geq 1.5 \mathrm{mmol} / \mathrm{L} \text { in women). } \neq \text { Diagnosis of hypertriglyceridemic waist is binary (presence or } \\
\text { absence), so the diagnosis cannot be used as a continuous variable to compute its correlation with VAT; however, hypertriglyceridemic waist is associated with a } 75-85 \% \\
\text { probability of increased levels of VAT. }\end{array}$} \\
\hline
\end{tabular}

suggest that a reduction in waist circumference is associated with a reduction in VAT, precise estimation of individual VAT reduction from waist circumference is not possible. Nonetheless, the corresponding reduction of VAT with waist circumference in a dose-dependent manner highlights the importance of routine measurement of waist circumference in clinical practice. Although no consensus exists regarding the best protocol for the measurement of waist circumference, many experts have recommended measurement at the level of the iliac crest, or at the midpoint between the last rib and the iliac crest. ${ }^{46}$

\section{Abdominal SAT and VAT: contribution to health outcomes}

The ability to distinguish previously externally undetectable inner body-fat compartments from the SAT that is externally visible affords investigators the opportunity to quantify the relations between various body fat depots and health outcomes. Early imaging work done in the 1980s revealed that the amount of intra-abdominal adipose tissue or VAT seemed to be the strongest adipose correlate of metabolic abnormalities that had been previously attributed to an excess amount of total body fat. ${ }^{47-49}$ There was, however, an initial confusion in the interpretation of these early data, which largely arose from a report showing that the respective correlations between SAT and VAT and metabolic outcomes were not substantially different, ${ }^{50}$ leading some investigators to suggest that subcutaneous fat was more important to consider than was visceral adiposity and that information on visceral adiposity would not substantially improve our ability to identify high-risk patients with overweight or obesity. This problem can be explained by the fact that, at the population level, there is a significant correlation between the sizes of SAT and VAT. ${ }^{37}$
However, studies that have quantified individual variation in visceral adiposity have identified a variation of two to three times in visceral adiposity at any level of total or subcutaneous adiposity. ${ }^{3,16,37}$ These observations emphasise the physiological role of SAT as the metabolic sink for excess triglycerides, albeit with a finite expansion capacity; thus, studies in rodents and human beings ${ }^{51,52}$ have shown that when SAT is unable to expand to accommodate overfeeding, visceral and ectopic fat deposition occurs. The hypothesis that the plasticity and expandability of SAT (or lack thereof) protects (or predisposes) individuals from visceral and ectopic fat deposition and subsequent metabolic health consequences led to the concept of a socalled personal fat threshold, ${ }^{51}$ which, if exceeded, increases the likelihood of developing metabolic disturbances such as type 2 diabetes, even when overall BMI is in the normal or mildly overweight range. ${ }^{52}$

Thus, to better understand whether measurement of visceral adiposity is useful or not to discriminate health risk, whether variation in visceral adiposity is associated with differences in the metabolic profile must be assessed within given levels of subcutaneous (or total) adiposity. Studies in which such analyses have been done $e^{14,53,54}$ have clearly shown that, within categories of total body or subcutaneous fat, high levels of VAT are associated with disturbances in the metabolic profile. An extensive discussion of the constellation of metabolic abnormalities associated with a selective excess of VAT is beyond the scope of this position statement, but several reviews on the topic have been published over the past 25 years. . $^{13,1,4,7,55-58}$

Many health and metabolic abnormalities are associated with an excess of VAT (figure 2). Type 2 diabetes is one such health consequence that might be reversed to normal glucose tolerance by substantial weight or VAT
Correspondence to: Prof Jean-Pierre Després, Department of Kinesiology, Faculty of Medicine, Université Laval, Quebec Heart and Lung Institute, Quebec City, QC G1V 4G5, Canada jean-pierre.despres@criucpq. ulaval.ca 


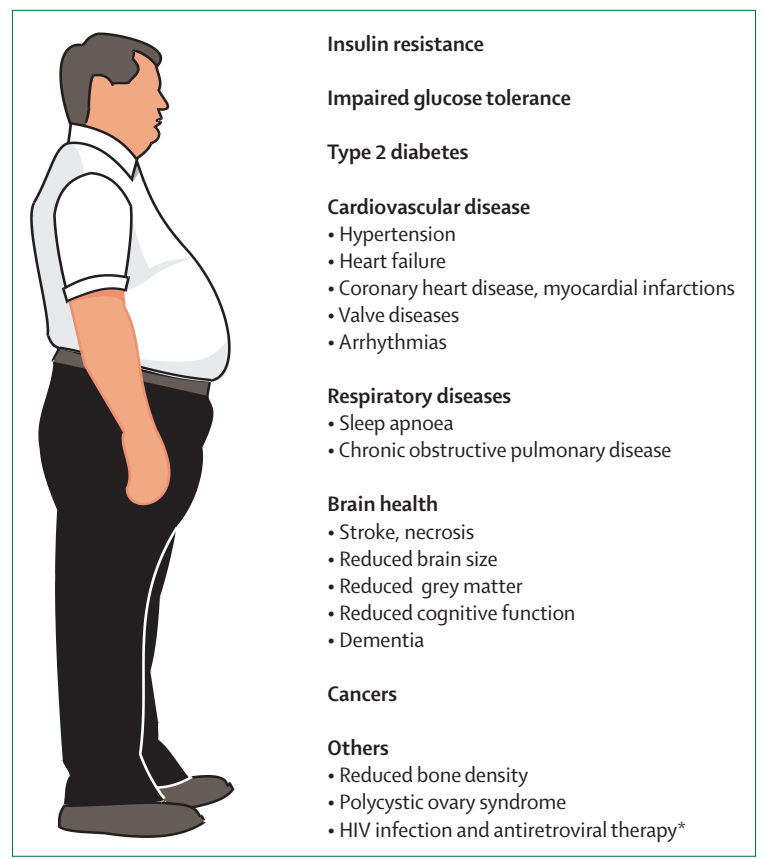

Figure 2: Health and metabolic abnormalities associated with an excess of visceral adipose tissue

Figure shows a list of clinical outcomes that have been reported to be associated with an excessive accumulation of visceral adipose tissue and ectopic fat irrespective of total adiposity estimated by BMI. For some of these conditions, evidence for a causal relation is insufficient. *HIV infection and antiretroviral therapy can contribute to the accumulation of visceral adipose tissue and ectopic fat.

loss (whether by diet, pharmacotherapy, or surgery). For example, in early type 2 diabetes, a negative calorie balance decreases liver fat and normalises regulation of hepatic gluconeogenesis..$^{59}$ Over the subsequent weeks, a continued caloric deficit improves intra-pancreatic fat levels and normalises first-phase insulin secretion..$^{59}$ Even in the long-term (up to 5 years), among people who undergo weight loss with bariatric surgery, BMI is lowered by $20 \%$, and $23-29 \%$ of patients are able to achieve an $\mathrm{HbA}_{1 \mathrm{c}}$ level of $6 \cdot 0 \%(42.1 \mathrm{mmol} / \mathrm{mol})$ or less with or without diabetes medications, compared with $5 \%$ among those treated with intensive medical therapy alone. ${ }^{60}$ Notably, even among individuals without obesity, targeted fat reduction can improve glycaemic control; this observation is consistent with the concept of the personal fat threshold. ${ }^{52}$

\section{Pathophysiology of visceral and ectopic fat}

Growing evidence suggests that VAT is a key conduit through which obesity predicts health risk. Visceral adiposity can be described as a consequence of a pathological response of SAT to positive caloric balance (inability of SAT to expand through hyperplasia of preadipocytes) in susceptible individuals, contributing directly and indirectly to cardiovascular and metabolic diseases. Excess visceral adiposity is characterised by increased visceral and ectopic fat deposition, adipocyte dysfunction, inflammatory and adipokine dysregulation, and insulin resistance. Multiple factors might influence fat deposition in various depots, including age, sex, race or ethnic origin, genetics and gene expression, the potential for adipose tissue expansion (hyperplasia vs hypertrophy), and response to weight gain.,13

The mechanisms by which an excess of VAT is related to various health outcomes are not fully understood and are under investigation. Several comprehensive reviews have been published on the topic ${ }^{3,55,61}$ and only a brief summary is provided here. Three non-mutually exclusive scenarios have been proposed to explain the cardiometabolic complications found among individuals with an excess of VAT: first, that VAT has metabolic properties that are distinct from SAT; second, that excess VAT induces inflammation; and third, that VAT is a marker of increased ectopic fat.

\section{VAT and SAT have distinct metabolic properties}

Because most VAT is drained by the portal vein, the hyperlipolytic state of large (hypertrophic) adipocytes associated with visceral obesity exposes the liver to high concentrations of free fatty acids and glycerol, leading to several impairments in liver metabolism, such as reduced hepatic extraction of insulin (exacerbating hyperinsulinemia), and increased production of triglyceriderich lipoproteins, as well as increased production of hepatic glucose, which explains the link between visceral obesity and glucose intolerance and type 2 diabetes. ${ }^{62}$ Free fatty acids have been shown to serve as ligands for peroxisome proliferator-activated receptor- $\alpha$ and hepatic nuclear factor in the liver and are involved in the gene transcription of several enzymes. For example, increased influx of free fatty acids into the liver has been shown to induce enhanced gene transcription of hepatic acylcoenzyme A synthetase, one of the limiting enzymes of triglyceride synthesis, as well as of microsomal triglyceride transfer protein (a rate limiting protein involved in the secretion of VLDLs), contributing to the development of hypertriglyceridemia. ${ }^{63}$ Visceral fat accumulation is also associated with the enhanced expression of aquaporin adipose, an adipocyte-specific glycerol channel, which results in increased glycerol release from VAT. ${ }^{64}$ This increased glycerol release is taken up by the liver through a liver-specific aquaporin, AQP-9, and converted into glycerol phosphate by glycerokinase and other enzymes as one of the mechanisms contributing to hyperglycaemia..$^{63-65}$

\section{Excess VAT induces inflammation}

The expanded VAT becomes inflamed because of the infiltration of macrophages among hypertrophied adipocytes, leading to increased production of inflammatory cytokines (such as tumour necrosis factor $\alpha$ and interleukin 6) and reduced production of a protective adipokine, adiponectin. Adiponectin is an anti-atherogenic, anti-diabetic, and anti-inflammatory protein, which is abundantly released from both SAT and 
VAT, but excess VAT is associated with the decreased production of adiponectin in whole-body adipose tissues, probably because of the increased secretion of inflammatory adipokines. ${ }^{66}$ Dietary omega-3 long-chain polyunsaturated fatty acids have been shown to modify visceral and ectopic fat and inflammatory mediators in obese rats. ${ }^{67}$ An extensive discussion of the emerging interplay between omega-3 fatty acids, ectopic adipose tissue, inflammation, and immunometabolism is beyond the scope of this position statement, but has been extensively reviewed elsewhere. ${ }^{68}$

\section{VAT is a marker of increased ectopic fat}

In response to excess triglycerides, the insufficient ability of SAT to expand through hyperplasia and to act as a protective metabolic sink in the context of a positive energy balance (resulting from excessive caloric intake, reduced energy expenditure due to physical inactivity, or a combination of these two components of the energy balance equation) causes lipid deposition in tissues that are not otherwise designed for adipose storage. This lipid spillover leads to lipid accumulation in normally lean tissues such as the liver, the heart (pericardial, epicardial, and intramyocardial), and skeletal muscle-a process known as ectopic fat deposition. In this context, excess visceral adiposity is a hallmark feature of dysfunctional SAT, a concept now widely recognised in the field of regional body fat distribution. ${ }^{69,70}$

Ectopic fat and health risk: marker or mediator?

There is an unresolved debate that is clinically relevant about the respective contributions of various ectopic fat depots (eg, liver, heart, muscle, pancreas, and kidney) to the dysmetabolic state and health complications associated with an excess of VAT. Perhaps the most hotly debated issue concerns liver fat. There are robust data showing that excess liver fat (for which non-alcoholic fatty liver disease [NAFLD] is a common clinical manifestation) is associated with most of the metabolic and inflammatory complications of visceral obesity. ${ }^{7174}$ For example, in one study, ${ }^{75}$ a high sugar diet altered the VLDL kinetics such that there were statistically significant differences in the production and catabolic rates of VLDL subclasses between men with NAFLD and controls, in response to the high and low sugar diets. Men with NAFLD had higher plasma concentrations of VLDL1-triacylglycerol (TAG) after the high and low sugar diets, a lower VLDL1TAG fractional catabolic rate after the high sugar diet, and a higher VLDL1-TAG production rate after the low sugar diet, than did controls. In that study, there were small changes in bodyweight, with both NAFLD and control groups gaining approximately $2 \mathrm{~kg}$ of bodyweight on the high sugar diet and losing approximately $2 \mathrm{~kg}$ on the low sugar diet. These small but statistically significant changes in bodyweight were related to changes in liver fat (measured by magnetic resonance spectroscopy [MRS]) but not to changes in VAT. These findings represent a situation in which diet can affect serum lipids and lipoprotein kinetics through weight-related or energyrelated effects on liver fat, but these effects are independent of VAT. On the basis of these and other data, some investigators have suggested that most of the metabolic complications of visceral obesity are mediated by the metabolic derangements that accompany excess liver fat. ${ }^{76,77}$ However, whether excess liver fat in isolation (ie, in the absence of excess VAT) is as detrimental as visceral obesity is not clear. Furthermore, the prevalence of NAFLD in isolation (without visceral obesity) is not well established.

Prospective observational studies have linked the presence of NAFLD with an increased risk of type 2 diabetes $^{78}$ and cardiovascular disease. ${ }^{79}$ However, these observations are limited by heterogeneity with respect to how NAFLD was diagnosed (via different imaging techniques - eg, ultrasound, CT, or MRS — tests for altered liver enzymes, and [rarely] liver biopsy), by the limited ability to grade liver disease severity in epidemiological studies (steatosis to steato-hepatitis or non-alcoholic steatohepatitis [NASH] to fibrosis), and by the presence of concomitant deposits of visceral fat and their consequent metabolic derangements.

One possibility to explore the isolated role of excess liver fat on cardiometabolic risk is to test effects of genetic variants that predispose to cardiometabolic risk without the concomitance of other excess fat accumulation. Studies that tested the PNPLA3 I148M variant, which commonly predisposes to NAFLD, showed inconsistent associations with incidence of major cardiovascular events. ${ }^{80,81}$ The TM6SF2 E167K variant also increases liver fat $^{82}$ and induces NASH and liver fibrosis ${ }^{83}$ by reducing VLDL secretion and liver triglyceride export; yet, individuals with this variant have NAFLD but low concentrations of apolipoprotein B-containing lipoproteins in the blood. A Mendelian randomisation study done in a Danish population ${ }^{80}$ did not establish a causal association between NAFLD and cardiovascular disease by testing variants in the TM6SF2 gene. A metaanalysis done as part of the same investigation, ${ }^{80}$ which included data from the Danish population and the CARDIoGRAMplusC4D consortium, reached similar conclusions.

An important clinical caveat of the concept of NALFD independently predisposing to increased cardiometabolic risk is that there is no evidence that its diagnosis will further add to risk stratification and subsequent management in addition to usual measures of excess adiposity, such as BMI and waist circumference. Certainly, from the hepatologist's perspective, the association of NAFLD with hepatic complications such as fibrosis, cirrhosis, and hepatocellular carcinoma needs to be seriously considered, and there is an unmet need for treatments that reduce liver fat accumulation and NASH.$^{84}$ Such treatments might also have the potential to mitigate downstream cardiometabolic risk, although 
prospective studies will be needed to investigate this possibility.

The relative contributions of the various ectopic fat depots to the risk of complications might depend on the health outcome considered, with some depots having local effects and others contributing to systemic effects (or both). ${ }^{17,73,85}$ For example, excess pericardial and epicardial fat is associated with atherosclerotic cardiovascular disease ${ }^{86}$ and atrial fibrillation. ${ }^{87}$ The close proximity of the ectopic fat depot to the coronary arteries and atrial myocardium (and conduction system) might predispose to cardiac-specific health risks because of local, paracrine, effects of lipotoxicty. ${ }^{8}$ However, excess pericardial and epicardial fat in isolation is much less common than its form associated with visceral obesity, making it difficult to determine its independent contribution to cardiac outcomes. For now, excess visceral adiposity could represent a useful indicator of an atherogenic and diabetogenic pattern of body fat distribution and a marker of dysfunctional SAT, making visceral adiposity an important target for diagnosis and treatment.

\section{Do visceral and ectopic fat respond to treatment?}

Evidence from several reviews and meta-analyses suggests that a decrease in energy intake or an increase in energy expenditure is associated with a reduction in VAT and SAT ${ }^{89-96}$ A meta-analysis by Sabag and colleagues,${ }^{95}$ showed that aerobic type exercise was associated with a significant reduction in VAT in adults with type 2 diabetes. Similarly, Verheggen and colleagues ${ }^{96}$ did a meta-analysis wherein eight studies compared a hypocaloric diet with exercise. Although both strategies were associated with significant reductions in VAT, a 5\% loss in bodyweight was associated with a $21 \%$ reduction in VAT after exercise, whereas only a $13 \%$ reduction in VAT was observed with a hypocaloric diet. A review by Hammond and colleagues ${ }^{90}$ concludes that exercise of moderate to high intensity $\left(50-70 \% \mathrm{VO}_{2}\right.$ peak) in adults is associated with substantial reductions in VAT and SAT.

Several randomised controlled trials have assessed whether the reduction in abdominal fat depots in adults differs in response to diet-induced or exercise-induced weight loss when the negative energy balance induced by diet or exercise is matched..$^{97,98}$ Irrespective of sex, weight loss of about $7 \%$ was associated with a reduction in VAT of about $25 \%$ in response to diet or exercise. These observations are consistent with the review of Verheggen and colleagues, ${ }^{96}$ wherein the results from eight studies in adults showed that diet and exercise are associated with similar reductions in VAT when the negative energy balance is matched. Irrespective of age, sex, or race, a negative energy balance induced by diet or exercise is generally associated with reductions in both VAT and SAT. ${ }^{90,91,93,99}$ However, the response of VAT and SAT depends on how their changes are expressed. If the reduction in either depot is expressed in absolute measures (eg, reduction in $\mathrm{cm}^{3}$ ), generally men lose more VAT than do women, because men have more VAT at baseline. ${ }^{96,100}$ Similarly, because women generally have more abdominal SAT than men, the reduction in SAT $\left(\mathrm{cm}^{3}\right)$ in response to diet or exercise is greater in women than it is in men. However, if changes in SAT or VAT are expressed relative to baseline measures (eg, percentage reduction), the reduction in both adipose tissue depots is similar in men and women.

The evidence is mixed on the issue of whether a doseresponse relation exists between exercise and reduction in VAT and SAT. ${ }^{41,42,90,93,100,101}$ Studies that have examined the effect of exercise dose generally support the conclusion that increasing exercise-induced energy expenditure is positively associated with corresponding reductions in VAT and SAT. ${ }^{42,90,94}$ Of particular interest to clinicians, several studies have shown clinically significant VAT reduction in response to exercise in the absence of substantial weight loss.91,96 Gepner and colleagues reported the results of an 18-month randomised controlled trial among 278 sedentary adults with abdominal obesity or dyslipidaemia in a single workplace setting in Israel. ${ }^{102}$ Participants were randomly assigned to a Mediterranean and low-carbohydrate diet (plus $28 \mathrm{~g}$ walnuts per day) or an isocaloric low-fat diet, with or without added moderate physical activity (80\% aerobic; supervised with free gym membership). 240 (86\%) participants completed the trial but all participants were included in the intention to treat analysis. Although both groups had similar moderate weight loss, VAT (22\%), intrahepatic (29\%), and intrapericardial (11\%) fat losses were greater than pancreatic and femur intermuscular fat losses (1-2\%). The Mediterranean and low-carbohydrate diet was better than the low-fat diet in decreasing intrahepatic, intrapericardial, and pancreatic fats; the addition of physical activity to either diet led to a significant decrease in VAT (mean difference $-6.67 \mathrm{~cm}^{2}$, $95 \%$ CI -14.8 to $-0 \cdot 45$ ), independent of weight loss. This trial provides robust evidence that moderate weight loss alone inadequately reflects the important effects of lifestyle interventions on atherogenic and diabetogenic fat depots, the Mediterranean diet seems to mobilise specific ectopic fat depots, and exercise independently contributes to VAT loss.

Although the evidence clearly establishes that regular exercise combined with a healthy diet is associated with a substantial reduction in VAT independent of age, sex, and ethnic origin, ${ }^{42,103,104}$ the separate effects of exercise amount and intensity on VAT are less clear. Findings from systematic reviews suggest that exercise amountenergy expenditure defined by metabolic equivalent of task-h per week ${ }^{104}$ or $\mathrm{kcals}^{42}$-is positively associated with reductions in VAT. ${ }^{104}$ However, the findings from the few randomised trials specifically designed to examine the independent contributions of exercise-induced energy expenditure on VAT report findings to the contrary-that 
an increase in exercise amount is not positively associated with VAT reduction. In a trial of inactive adults with overweight or obesity, Keating and colleagues reported that exercise conditions varying in total time resulted in VAT reductions of a similar magnitude. ${ }^{105}$ Similarly, in another trial, Slentz and colleagues reported that there was no difference in VAT reduction between groups that vary in exercise amount among adults with obesity. ${ }^{106}$ These observations were supported by findings from the trial by Cowan and colleagues, who reported that increasing exercise amount without changes in energy intake for 6 months was not associated with increased VAT reduction in adults with abdominal obesity. ${ }^{107}$

Whether exercise intensity is associated with VAT reduction independent of exercise amount is also unclear. Whereas the primary finding from a review that compared exercise groups that varied in exercise intensity across studies suggests that intensity is positively associated with reductions in VAT, ${ }^{100}$ preliminary findings from randomised trials are inconclusive. Similar to observations for exercise amount, Keating and colleagues, Slentz and colleagues, Cowan and colleagues, and Irving and colleagues reported no statistically significant effect of increasing exercise intensity on VAT reduction..$^{105-08}$

Cowan and colleagues ${ }^{107}$ considered whether the separate effects of exercise amount and intensity on VAT reduction differed across the abdominal region by analysing the change in VAT for five magnetic resonance images, extending from below to above L4-5 (figure 3). The two major observations from this analysis were that a substantial reduction in VAT occurs in response to exercise combined with a balanced diet (ie, participants do not eat more food to compensate for energy lost via exercise) irrespective of amount or intensity, and that the reduction in VAT is uniform across the abdomen. ${ }^{107}$

In summary, exercise and dietary interventions are associated with reduction in VAT, irrespective of amount or intensity of exercise. A weight loss of about $5 \%$ resulting from a lifestyle-based strategy consistent with current recommendations is likely to result in a 15-25\% reduction in VAT..$^{00,96,97,98}$ These observations provide treatment options to target VAT reduction as part of a comprehensive lifestyle-based strategy to reduce health risk.

Although intervention studies have shown that loss of VAT is associated with cardiometabolic benefits among individuals with excess visceral adiposity, public health education and clinical approaches still focus on assessing the risk of obesity by BMI and on assessing the success of its management by the magnitude of weight loss achieved. In Japan, investigators of the longitudinal Amagasaki Visceral Fat Study have explored the relevance of classifying individuals not only on the basis of their BMI but also on the basis of their estimated level of VAT. ${ }^{109}$ Several reports have been published on this study. ${ }^{109-11}$ Overall, findings indicate that changes in weight and VAT observed (no change or



Figure 3: Regional differences in VAT reduction across the abdomen in response to exercise of varying amounts and intensities

Data shown are mean values and error bars are the associated standard error of the means. The MRI measures were taken before and after intervention (24 weeks). Absolute $\left(\mathrm{cm}^{3} ; \mathrm{A}\right)$ and relative (\%; B) changes in VAT are shown at the L4-5 intervertebral space; $5 \mathrm{~cm}$ below L4-5; and $5 \mathrm{~cm}, 10 \mathrm{~cm}$, and $15 \mathrm{~cm}$ above L4-5. Total VAT change (the sum of each individual slice) is also shownfor absolute change, total change is shown in kg; for relative change, total changes are percentage values. Reproduced from Cowan and colleagues, ${ }^{107}$ by permission of Wiley-Blackwell on behalf of The Obesity Society. VAT=visceral adipose tissue. . Control=no exercise. $\mathrm{LALI}=$ low amount, low-intensity exercise (180 kcal/session for women and $300 \mathrm{kcal} / \mathrm{session}$ for men, at $50 \% \mathrm{VO}_{2}$ peak). $\mathrm{HALI}=$ high amount, low-intensity exercise $(360 \mathrm{kcal} / \mathrm{session}$ for women and $600 \mathrm{kcal} /$ session for men, at $50 \% \mathrm{VO}_{2}$ peak). $\mathrm{HAHI}=$ high amount, high-intensity exercise (360 for $\mathrm{kcal} / \mathrm{session}$ women and $600 \mathrm{kcal} / \mathrm{session}$ for men, at $75 \% \mathrm{VO}_{2}$ peak).

increase vs decrease) in response to a health promotion and education programme focussing on the importance of VAT were associated with corresponding changes (deterioration or improvement) in variables reflecting cardiometabolic risk. Also, individuals who initially had the highest level of VAT for their BMI benefited the most from the health promotion programme. Although this longitudinal study was not powered to compare cardiovascular disease outcomes between participants who lost VAT versus those who gained VAT, investigators found a lower cumulative incidence of cardiovascular outcomes among participants who lost VAT compared with those who gained VAT over the 50-month followup of the study. ${ }^{109}$ Although these findings cannot quantify the respective contributions of weight loss versus loss of VAT, they highlight the potential relevance of monitoring visceral adiposity in cardiometabolic risk assessment and management. Large-scale cardiometabolic imaging studies that are underway should help to address this question. 


\section{Conclusions}

Although the link between BMI and morbidity and mortality risk is firmly established at the population level, considerable variation exists at the individual level in the cardiometabolic risk profile in people with the same BMI. Many findings from CT and MRI studies have shown the limitations of BMI as a clinical tool to identify overweight and obese individuals with excess VAT, who represent the subgroup of individuals at highest cardiometabolic risk. Additionally, at any BMI value, excess visceral adiposity is often associated with an increased accumulation of lipids in normally lean tissues such as the heart, liver, and skeletal muscle, a harmful condition referred to as ectopic fat deposition.

Despite clear and convincing evidence that the accumulation of visceral and ectopic fat is a major contributor to cardiovascular disease and metabolic risk above and beyond BMI, the assessment of fat distribution in clinical practice remains a challenge. There is therefore a need to develop methods to identify individuals with excess visceral adiposity and ectopic fat in clinical practice and in public health programmes that would go beyond tracking and targeting BMI at the population level. Since VAT is the most diabetogenic and atherogenic fat depot, there is a need to develop simple, clinically applicable tools to be able to monitor changes in VAT and ectopic fat over time. Addition of the measurement of plasma triglyceride concentrations to the measurement of waist circumference (to identify hypertriglyceridemic waist) ${ }^{34}$ might be helpful, but more refined tools that are validated with imaging data should be developed to further refine the definition of high-risk overweight and obesity.

\section{Search strategy and selection criteria}

This position statement is a narrative review designed to provide a consensus perspective of the International Atherosclerosis Society and International Chair on Cardiometabolic Risk Working Group on Visceral Obesity. First, the group initially had a face-to-face meeting on April 24, 2017, to review the high-quality evidence available known to the subject experts. No formal comprehensive literature search was done because this Review is a joint consensus position statement rather than a formal literature review. After discussion and deliberation among the group regarding the context and quality of the evidence, an executive writing group was formed and was responsible for writing the first draft. This draft was then circulated to all authors for critical revision of intellectual content pertinent to each authors' expertise. High-quality published literature that became available after the initial face-to-face meeting (until Dec 31, 2018) was identified by all authors and reviewed by the executive writing group for inclusion in the manuscript. The first author coordinated the final preparation and submission of the position statement after the group achieved consensus and approved its content.
Anthropometric indices of obesity are easily implemented, but newer imaging-based methods offer improved sensitivity and specificity for measuring specific depots. Lifestyle, pharmacologic, and surgical interventions permit a multidisciplinary approach to target visceral and ectopic fat that might improve outcomes. Finally, researchers and clinicians need to align the evidence with a public health message to focus on visceral and ectopic fat in addition to excess bodyweight to better combat the growing epidemic of obesity worldwide.

\section{Contributors}

This position statement reflects the consensus position of the International Atherosclerosis Society and International Chair on Cardiometabolic Risk Working Group on Visceral Obesity. The group initially had a face-to-face meeting to review the evidence available and all authors provided input regarding the content of the position statement. An executive writing group consisting of IJN, RR, RDS, J-PD, JS, IS, SY, and YM was responsible for writing the first draft. The original draft was then circulated to all authors for critical revision of intellectual content. IJN coordinated the final preparation and submission of the position statement after the group achieved consensus and approved its content.

\section{Declaration of interests}

IJN reports fees for consulting and serving on advisory boards from Boehringer Ingelheim-Lilly Alliance and AMRA Medical and a research grant from Novo Nordisk. FBH reports speaker fees from Metagenics and Standard Process and a research grant from the California Walnut Commission. RDS reports consulting and speaker fees from Amgen, AstraZeneca, Biolab, Merck, Novo Nordisk, Sanofi Regeneron, Akcea, Kowa, and Esperion. SY reports research grants from Kowa Company, Otsuka Pharmaceutical Co, Shionogi and Co, Bayer Yakuhin, MSD KK, Takeda Pharmaceutical Company, Sanwa Kagaku Kenkyusho Co, Astellas Pharma, Daiichi Sankyo, AstraZeneca KK, Kaken Pharmaceutical Co, Nippon Boehringer Ingelheim Co, the Japanese National Institute of Biomedical Innovation, Kyowa Medex, Mochida Pharmaceutical Company, Hayashibara Co, Teijin Pharma, Pfizer, and Kissei; speaker fees from Kowa Company, MSD KK, Bayer Yakuhin, Amgen Astellas BioPharma, Astellas Pharma, Sanofi, Otsuka Pharmaceutical Co, Ono Pharmaceutical Company, and Aegerion; and serving on the advisory board of Skylight Biotech. SY has patents issued by Fujirebio (PCT/JP2016/074402, Assisting Method for the Diagnosis of Type III Hyperlipidemia) and Kyowa Medex Co (PCT/ JP2017/038715, Method for Measuring Oxidized High-Density Lipoprotein; PCT/JP2017/038766, Method for Selecting Subject Needing Treatment for Dyslipidemia and Reagent for Such Selection). All other authors declare no competing interests.

\section{Acknowledgments}

The consensus meeting that led to the production of this position statement was held with the financial support of the International Atherosclerosis Society (IAS). The International Chair on

Cardiometabolic Risk (ICCR), an independent academic organisation based at Université Laval (Quebec, QC, Canada), was responsible for coordinating the production of the position statement, which reflects the joint position of the IAS-ICCR Working Group on Visceral Obesity. No funding or honoraria were provided by either IAS or the ICCR to the members of the Working Group to produce this position statement.

\section{References}

1 GBD 2015 Obesity Collaborators. Health effects of overweight and obesity in 195 countries over 25 years. N Engl J Med 2017; 377: $13-27$

2 Benjamin EJ, Virani SS, Callaway CW, et al. Heart disease and stroke statistics-2018 update: a report from the American Heart Association. Circulation 2018; 137: e67-492.

3 Tchernof A, Després JP. Pathophysiology of human visceral obesity: an update. Physiol Rev 2013; 93: 359-404.

4 Gonzalez-Muniesa P, Martinez-Gonzalez MA, Hu FB, et al. Obesity. Nat Rev Dis Primers 2017; 3: 17034 
5 Wildman RP, Muntner P, Reynolds K, et al. The obese without cardiometabolic risk factor clustering and the normal weight with cardiometabolic risk factor clustering: prevalence and correlates of 2 phenotypes among the US population (NHANES 1999-2004). Arch Intern Med 2008; 168: 1617-24.

6 Després JP. What is "metabolically healthy obesity"?: from epidemiology to pathophysiological insights. J Clin Endocrinol Metab 2012; 97: 2283-85.

7 Mongraw-Chaffin M, Foster MC, Anderson CAM, et al. Metabolically healthy obesity, transition to metabolic syndrome, and cardiovascular risk. J Am Coll Cardiol 2018; 71: 1857-65.

8 Bays HE, McCarthy W, Christensen S, et al. Obesity algorithm slides, presented by the Obesity Medicine Association. 2019. https:// obesitymedicine.org/obesity-algorithm/ (accessed June 7, 2019).

9 Brauer P, Connor Gorber S, Shaw E, et al. Recommendations for prevention of weight gain and use of behavioural and pharmacologic interventions to manage overweight and obesity in adults in primary care. CMAJ 2015; 187: 184-95.

10 Garvey WT, Mechanick JI, Brett EM, et al. American Association of Clinical Endocrinologists and American College of Endocrinology comprehensive clinical practice guidelines for medical care of patients with obesity. Endocr Pract 2016; 22 (suppl 3): 1-203.

11 Jensen MD, Ryan DH, Apovian CM, et al. 2013 AHA/ACC/TOS guideline for the management of overweight and obesity in adults: a report of the American College of Cardiology/American Heart Association Task Force on Practice Guidelines and The Obesity Society. Circulation 2014; 129 (25 suppl 2): S102-38.

12 Tsigos C, Hainer V, Basdevant A, et al. Management of obesity in adults: European clinical practice guidelines. Obes Facts 2008; 1: 106-16.

13 Neeland IJ, Poirier P, Després JP. Cardiovascular and metabolic heterogeneity of obesity: clinical challenges and implications for management. Circulation 2018; 137: 1391-406.

14 Fox CS, Massaro JM, Hoffmann U, et al. Abdominal visceral and subcutaneous adipose tissue compartments: association with metabolic risk factors in the Framingham Heart Study. Circulation 2007; 116: 39-48.

15 Neeland IJ, Ayers CR, Rohatgi AK, et al. Associations of visceral and abdominal subcutaneous adipose tissue with markers of cardiac and metabolic risk in obese adults. Obesity (Silver Spring) 2013; 21: e439-47.

16 Hiuge-Shimizu A, Kishida K, Funahashi T, et al. Absolute value of visceral fat area measured on computed tomography scans and obesity-related cardiovascular risk factors in large-scale Japanese general population (the VACATION-J study). Ann Med 2012; 44: 82-92.

17 Després JP. Body fat distribution and risk of cardiovascular disease: an update. Circulation 2012; 126: 1301-13.

18 Borkan GA, Gerzof SG, Robbins AH, Hults DE, Silbert CK, Silbert JE. Assessment of abdominal fat content by computed tomography. Am J Clin Nutr 1982; 36: 172-77.

19 Sjöström L, Kvist H, Cederblad A, Tylén U. Determination of total adipose tissue and body fat in women by computed tomography, 40K, and tritium. Am J Physiol 1986; 250 (6 pt 1): e736-45.

20 Tokunaga K, Matsuzawa Y, Ishikawa K, Tarui S. A novel technique for the determination of body fat by computed tomography. Int J Obes 1983; 7: 437-45.

21 Heymsfield SB, Gonzalez MC, Lu J, Jia G, Zheng J. Skeletal muscle mass and quality: evolution of modern measurement concepts in the context of sarcopenia. Proc Nutr Soc 2015; 74: 355-66.

22 Lee JJ, Pedley A, Hoffmann U, Massaro JM, Fox CS. Association of changes in abdominal fat quantity and quality with incident cardiovascular disease risk factors. J Am Coll Cardiol 2016; 68: 1509-21.

23 Arsenault BJ, Beaumont EP, Després JP, Larose E. Mapping body fat distribution: a key step towards the identification of the vulnerable patient? Ann Med 2012; 44: 758-72.

24 Mathieu P, Boulanger MC, Després JP. Ectopic visceral fat: a clinical and molecular perspective on the cardiometabolic risk. Rev Endocr Metab Disord 2014; 15: 289-98.

25 Linge J, Borga M, West J, et al. Body composition profiling in the UK Biobank imaging study. Obesity (Silver Spring) 2018; 26: 1785-95.
26 Kaul S, Rothney MP, Peters DM, et al. Dual-energy X-ray absorptiometry for quantification of visceral fat. Obesity (Silver Spring) 2012; 20: 1313-18.

27 Neeland IJ, Grundy SM, Li X, Adams-Huet B, Vega GL Comparison of visceral fat mass measurement by dual-X-ray absorptiometry and magnetic resonance imaging in a multiethnic cohort: the Dallas Heart Study. Nutr Diabetes 2016; 6: e221.

28 Gradmark AM, Rydh A, Renstrom F, et al. Computed tomography-based validation of abdominal adiposity measurements from ultrasonography, dual-energy X-ray absorptiometry and anthropometry. Br J Nutr 2010; 104: 582-88.

29 Pouliot MC, Després JP, Lemieux S, et al. Waist circumference and abdominal sagittal diameter: best simple anthropometric indexes of cardiovascular risk in men and women. Am J Cardiol 1994; 73: 460-68.

30 Neeland IJ, Eliason B, Ridderstrale M, et al. Comparison of adipose distribution indices with gold standard body composition assessments in the EMPA-REG H2H SU trial: a body composition sub-study. Diabetes Ther 2015; 6: 635-42.

31 Yang L, Samarasinghe YP, Kane P, Amiel SA, Aylwin SJ. Visceral adiposity is closely correlated with neck circumference and represents a significant indicator of insulin resistance in WHO grade III obesity. Clin Endocrinol (Oxf) 2010; 73: 197-200.

32 Preis SR, Massaro JM, Hoffmann U, et al. Neck circumference as a novel measure of cardiometabolic risk: the Framingham Heart study. J Clin Endocrinol Metab 2010; 95: 3701-10.

33 Lemieux I, Pascot A, Couillard C, et al. Hypertriglyceridemic waist: a marker of the atherogenic metabolic triad (hyperinsulinemia; hyperapolipoprotein B; small, dense LDL) in men? Circulation 2000; 102: $179-84$.

34 Lemieux I, Poirier P, Bergeron J, et al. Hypertriglyceridemic waist: a useful screening phenotype in preventive cardiology? Can J Cardiol 2007; 23 (suppl B): 23B-31B.

35 Ashwell M, Cole TJ, Dixon AK. Obesity: new insight into the anthropometric classification of fat distribution shown by computed tomography. Br Med J (Clin Res Ed) 1985; 290: 1692-94.

36 Snijder MB, van Dam RM, Visser M, Seidell JC. What aspects of body fat are particularly hazardous and how do we measure them? Int J Epidemiol 2006; 35: 83-92.

37 Nazare JA, Smith J, Borel AL, et al. Usefulness of measuring both body mass index and waist circumference for the estimation of visceral adiposity and related cardiometabolic risk profile (from the INSPIRE ME IAA study). Am J Cardiol 2015; 115: $307-15$

38 Grundy SM, Cleeman JI, Daniels SR, et al. Diagnosis and management of the metabolic syndrome: an American Heart Association/National Heart, Lung, and Blood Institute Scientific Statement. Circulation 2005; 112: 2735-52.

39 Alberti KG, Zimmet P, Shaw J. The metabolic syndrome-a new worldwide definition. Lancet 2005; 366: 1059-62.

40 Ross R, Janssen I, Dawson J, et al. Exercise-induced reduction in obesity and insulin resistance in women: a randomized controlled trial. Obes Res 2004; 12: 789-98.

41 Janiszewski PM, Ross R. Physical activity in the treatment of obesity: beyond body weight reduction. Appl Physiol Nutr Metab 2007; 32: 512-22.

42 Ross R, Janssen I. Physical activity, total and regional obesity: dose-response considerations. Med Sci Sports Exerc 2001; 33 (suppl 6): S521-27.

43 Janssen I, Heymsfield SB, Ross R. Application of simple anthropometry in the assessment of health risk: implications for the Canadian physical activity, fitness and lifestyle appraisal. Can J Appl Physiol 2002; 27: 396-414.

44 Ross R, Shaw KD, Rissanen J, Martel Y, de Guise J, Avruch L. Sex differences in lean and adipose tissue distribution by magnetic resonance imaging: anthropometric relationships. Am J Clin Nutr 1994; 59: 1277-85.

45 Villeneuve N, Pelletier-Beaumont E, Nazare JA, et al. Interrelationships between changes in anthropometric variables and computed tomography indices of abdominal fat distribution in response to a 1-year physical activity-healthy eating lifestyle modification program in abdominally obese men. Appl Physiol Nutr Metab 2014; 39: 503-11. 
46 Ross R, Berentzen T, Bradshaw AJ, et al. Does the relationship between waist circumference, morbidity and mortality depend on measurement protocol for waist circumference? Obes Rev 2008; 9: $312-25$.

47 Després JP, Moorjani S, Lupien PJ, Tremblay A, Nadeau A, Bouchard C. Regional distribution of body fat, plasma lipoproteins, and cardiovascular disease. Arteriosclerosis 1990; 10: 497-511.

48 Després JP, Nadeau A, Tremblay A, et al. Role of deep abdominal fat in the association between regional adipose tissue distribution and glucose tolerance in obese women. Diabetes 1989; 38: 304-09.

49 Fujioka S, Matsuzawa Y, Tokunaga K, Tarui S. Contribution of intra-abdominal fat accumulation to the impairment of glucose and lipid metabolism in human obesity. Metabolism 1987; 36: $54-59$.

50 Abate N, Garg A, Peshock RM, Stray-Gundersen J, Grundy SM. Relationships of generalized and regional adiposity to insulin sensitivity in men. J Clin Invest 1995; 96: 88-98.

51 Cuthbertson DJ, Steele T, Wilding JP, et al. What have human experimental overfeeding studies taught us about adipose tissue expansion and susceptibility to obesity and metabolic complications? Int J Obes (Lond) 2017; 41: 853-65.

52 Taylor R, Holman RR. Normal weight individuals who develop type 2 diabetes: the personal fat threshold. Clin Sci (Lond) 2015; 128: $405-10$

53 Ross R, Aru J, Freeman J, Hudson R, Janssen I. Abdominal adiposity and insulin resistance in obese men. Am J Physiol Endocrinol Metab 2002; 282: E657-63.

54 Ross R, Freeman J, Hudson R, Janssen I. Abdominal obesity, muscle composition, and insulin resistance in premenopausal women. J Clin Endocrinol Metab 2002; 87: 5044-51.

55 Després JP, Lemieux I. Abdominal obesity and metabolic syndrome. Nature 2006; 444: 881-87.

56 Després JP, Lemieux I, Bergeron J, et al. Abdominal obesity and the metabolic syndrome: contribution to global cardiometabolic risk. Arterioscler Thromb Vasc Biol 2008; 28: 1039-49.

57 Matsuzawa Y. Pathophysiology and molecular mechanisms of visceral fat syndrome: the Japanese experience. Diabetes Metab Rev 1997; 13: 3-13.

58 Matsuzawa Y, Funahashi T, Nakamura T. The concept of metabolic syndrome: contribution of visceral fat accumulation and its molecular mechanism. J Atheroscler Thromb 2011; 18: 629-39.

59 Taylor R. Calorie restriction and reversal of type 2 diabetes. Expert Rev Endocrinol Metab 2016; 11: 521-28.

60 Schauer PR, Bhatt DL, Kirwan JP, et al. Bariatric surgery versus intensive medical therapy for diabetes-5-year outcomes. N Engl J Med 2017; 376: 641-51.

61 Bays HE. Adiposopathy is "sick fat" a cardiovascular disease? J Am Coll Cardiol 2011; 57: 2461-73.

62 Neeland IJ, Hughes C, Ayers CR, Malloy CR, Jin ES. Effects of visceral adiposity on glycerol pathways in gluconeogenesis. Metabolism 2017; 67: 80-89.

63 Kuriyama H, Yamashita S, Shimomura I, et al. Enhanced expression of hepatic acyl-coenzyme A synthetase and microsomal triglyceride transfer protein messenger RNAs in the obese and hypertriglyceridemic rat with visceral fat accumulation. Hepatology 1998; 27: 557-62.

64 Kishida K, Kuriyama H, Funahashi T, et al. Aquaporin adipose, a putative glycerol channel in adipocytes. J Biol Chem 2000; 275: 20896-902.

65 Arita Y, Kihara S, Ouchi N, et al. Paradoxical decrease of an adipose-specific protein, adiponectin, in obesity. Biochem Biophys Res Commun 1999; 257: 79-83.

66 Ouchi N, Parker JL, Lugus JJ, Walsh K. Adipokines in inflammation and metabolic disease. Nat Rev Immunol 2011; 11: 85-97.

67 Batetta B, Griinari M, Carta G, et al. Endocannabinoids may mediate the ability of (n-3) fatty acids to reduce ectopic fat and inflammatory mediators in obese Zucker rats. J Nutr 2009. 139: $1495-501$

68 Kuda O, Rossmeisl M, Kopecky J. Omega-3 fatty acids and adipose tissue biology. Mol Aspects Med 2018; 64: 147-60.

69 Smith U, Kahn BB. Adipose tissue regulates insulin sensitivity: role of adipogenesis, de novo lipogenesis and novel lipids. J Intern Med 2016; 280: 465-75.
70 Vasan SK, Karpe F. Fat, yet fit. Nat Rev Endocrinol 2016; 12: 375-76.

71 Adiels M, Olofsson SO, Taskinen MR, Boren J. Overproduction of very low-density lipoproteins is the hallmark of the dyslipidemia in the metabolic syndrome. Arterioscler Thromb Vasc Biol 2008; 28: $1225-36$

72 Kotronen A, Yki-Jarvinen H. Fatty liver: a novel component of the metabolic syndrome. Arterioscler Thromb Vasc Biol 2008; 28: 27-38.

73 Ndumele CE, Nasir K, Conceicao RD, Carvalho JA, Blumenthal RS Santos RD. Hepatic steatosis, obesity, and the metabolic syndrome are independently and additively associated with increased systemic inflammation. Arterioscler Thromb Vasc Biol 2011; 31: 1927-32.

74 Tilg H, Moschen AR, Roden M. NAFLD and diabetes mellitus. Nat Rev Gastroenterol Hepatol 2017; 14: 32-42.

75 Umpleby AM, Shojaee-Moradie F, Fielding B, et al. Impact of liver fat on the differential partitioning of hepatic triacylglycerol into VLDL subclasses on high and low sugar diets. Clin Sci (Lond) 2017; 131: 2561-73.

76 Fabbrini E, Magkos F, Mohammed BS, et al. Intrahepatic fat, not visceral fat, is linked with metabolic complications of obesity. Proc Natl Acad Sci USA 2009; 106: 15430-35.

77 Klein S. Is visceral fat responsible for the metabolic abnormalities associated with obesity? Implications of omentectomy. Diabetes Care 2010; 33: 1693-94.

78 Valenti L, Bugianesi E, Pajvani U, Targher G. Nonalcoholic fatty liver disease: cause or consequence of type 2 diabetes? Liver Int 2016; 36: 1563-79.

79 Targher G, Byrne CD. Non-alcoholic fatty liver disease: an emerging driving force in chronic kidney disease. Nat Rev Nephrol 2017; 13: $297-310$

80 Lauridsen BK, Stender S, Kristensen TS, et al. Liver fat content, non-alcoholic fatty liver disease, and ischaemic heart disease: Mendelian randomization and meta-analysis of 279013 individuals. Eur Heart J 2018; 39: 385-93.

81 Posadas-Sanchez R, Lopez-Uribe AR, Posadas-Romero C, et al. Association of the I148M/PNPLA3 (rs738409) polymorphism with premature coronary artery disease, fatty liver, and insulin resistance in type 2 diabetic patients and healthy controls. The GEA study. Immunobiology 2016; 222: 960-66.

82 Kozlitina J, Smagris E, Stender S, et al. Exome-wide association study identifies a TM6SF2 variant that confers susceptibility to nonalcoholic fatty liver disease. Nat Genet 2014; 46: 352-56.

83 Dongiovanni P, Petta S, Maglio C, et al. Transmembrane 6 superfamily member 2 gene variant disentangles nonalcoholic steatohepatitis from cardiovascular disease. Hepatology 2015; 61: 506-14.

84 Eslam M, Valenti L, Romeo S. Genetics and epigenetics of NAFLD and NASH: clinical impact. J Hepatol 2018; 68: 268-79.

85 Britton KA, Fox CS. Ectopic fat depots and cardiovascular disease. Circulation 2011; 124: e837-41.

86 Mahabadi AA, Berg MH, Lehmann N, et al. Association of epicardial fat with cardiovascular risk factors and incident myocardial infarction in the general population: the Heinz Nixdorf Recall Study. J Am Coll Cardiol 2013; 61: 1388-95.

87 Wong CX, Sun MT, Odutayo A, et al. Associations of epicardial, abdominal, and overall adiposity with atrial fibrillation. Circ Arrhythm Electrophysiol 2016; 9: e004378.

88 Iacobellis G, Bianco AC. Epicardial adipose tissue: emerging physiological, pathophysiological and clinical features. Trends Endocrinol Metab 2011; 22: 450-57.

89 Chaston TB, Dixon JB. Factors associated with percent change in visceral versus subcutaneous abdominal fat during weight loss: findings from a systematic review. Int J Obes (Lond) 2008; 32: 619-28.

90 Hammond BP, Brennan AM, Ross R. Exercise and adipose tissue redistribution in overweight and obese adults. In: Lukaski HC, ed. Body composition: health and performance in exercise and sport. Boca Raton: CRC Press, 2017: 109-28.

91 Kay SJ, Fiatarone Singh MA. The influence of physical activity on abdominal fat: a systematic review of the literature. Obes Rev 2006; 7: 183-200.

92 Merlotti C, Ceriani V, Morabito A, Pontiroli AE. Subcutaneous fat loss is greater than visceral fat loss with diet and exercise, weight-loss promoting drugs and bariatric surgery: a critical review and meta-analysis. Int J Obes (Lond) 2017; 41: 672-82. 
93 Ohkawara K, Tanaka S, Miyachi M, Ishikawa-Takata K, Tabata I. A dose-response relation between aerobic exercise and visceral fat reduction: systematic review of clinical trials. Int J Obes (Lond) 2007; 31: 1786-97.

94 O'Neill T, Shalev-Goldman E, Ross R. Caloric restriction and/or increasing exercise in the management of obesity. In: Hansen D, ed. Exercise therapy in adult individuals with obesity. Hauppauge: Nova Science Publishers, 2013: 43-72.

95 Sabag A, Way KL, Keating SE, et al. Exercise and ectopic fat in type 2 diabetes: a systematic review and meta-analysis. Diabetes Metab 2017; 43: 195-210.

96 Verheggen RJ, Maessen MF, Green DJ, Hermus AR, Hopman MT, Thijssen DH. A systematic review and meta-analysis on the effects of exercise training versus hypocaloric diet: distinct effects on body weight and visceral adipose tissue. Obes Rev 2016; 17: 664-90.

97 Ross R, Dagnone D, Jones PJ, et al. Reduction in obesity and related comorbid conditions after diet-induced weight loss or exercise-induced weight loss in men. A randomized, controlled trial. Ann Intern Med 2000; 133: 92-103.

98 Brochu M, Malita MF, Messier V, et al. Resistance training does not contribute to improving the metabolic profile after a 6-month weight loss program in overweight and obese postmenopausal women. J Clin Endocrinol Metab 2009; 94: 3226-33.

99 Gasteyger C, Larsen TM, Vercruysse F, Pedersen D, Toubro S, Astrup A. Visceral fat loss induced by a low-calorie diet: a direct comparison between women and men. Diabetes Obes Metab 2009; 11: 596-602.

100 Vissers D, Hens W, Taeymans J, Baeyens JP, Poortmans J, Van Gaal L. The effect of exercise on visceral adipose tissue in overweight adults: a systematic review and meta-analysis. PLoS One 2013; 8: e56415.

101 Ross R, Hudson R, Stotz PJ, Lam M. Effects of exercise amount and intensity on abdominal obesity and glucose tolerance in obese adults: a randomized trial. Ann Intern Med 2015; 162: 325-34.

102 Gepner Y, Shelef I, Schwarzfuchs D, et al. Effect of distinct lifestyle interventions on mobilization of fat storage pools: CENTRAL magnetic resonance imaging randomized controlled trial. Circulation 2018; 137: 1143-57.
103 Thompson D, Karpe F, Lafontan M, Frayn K. Physical activity and exercise in the regulation of human adipose tissue physiology. Physiol Rev 2012; 92: 157-91.

104 Ohkawara K, Tanaka S, Miyachi M, Ishikawa-Takata K, Tabata I. A dose-response relation between aerobic exercise and visceral fat reduction: systematic review of clinical trials. Int J Obes (Lond) 2007; 31: 1786-97.

105 Keating SE, Hackett DA, Parker HM, et al. Effect of aerobic exercise training dose on liver fat and visceral adiposity. J Hepatol 2015; 63: $174-82$

106 Slentz CA, Aiken LB, Houmard JA, et al. Inactivity, exercise, and visceral fat. STRRIDE: a randomized, controlled study of exercise intensity and amount. J Appl Physiol (1985) 2005; 99: 1613-18.

107 Cowan TE, Brennan AM, Stotz PJ, Clarke J, Lamarche B, Ross R. Separate effects of exercise amount and intensity on adipose tissue and skeletal muscle mass in adults with abdominal obesity. Obesity (Silver Spring) 2018; 26: 1696-703.

108 Irving BA, Davis CK, Brock DW, et al. Effect of exercise training intensity on abdominal visceral fat and body composition. Med Sci Sports Exerc 2008; 40: 1863-72.

109 Okauchi Y, Kishida K, Funahashi T, et al. 4-year follow-up of cardiovascular events and changes in visceral fat accumulation after health promotion program in the Amagasaki Visceral Fat Study. Atherosclerosis 2010; 212: 698-700.

110 Ryo M, Nakamura T, Funahashi T, et al. Health education "hokenshido" program reduced metabolic syndrome in the Amagasaki Visceral Fat Study. Three-year follow-up study of 3174 Japanese employees. Intern Med 2011; 50: 1643-48.

111 Okauchi Y, Iwahashi H, Okita K, et al. Weight reduction is associated with improvement of glycemic control in Japanese men, whose hemoglobin A1C is 5.6-6.4\%, with visceral fat accumulation, but not without visceral fat accumulation. J Diabetes Investig 2013; 4: 454-59.

(C) 2019 Elsevier Ltd. All rights reserved. 